\title{
EPIDEMIOLOGIA DA HIPERTENSÃO ARTERIAL*
}

\author{
Cecília Amaro de Lolio**
}

\begin{abstract}
LOLIO, C. A. de. Epidemiologia da hipertensão arterial. Rev. Saúde públ., S. Paulo, 24:425-32, 1990.
RESUMO: E feita uma revisão dos conhecimentos atuais sobre a hipertensão arterial, com ênfase aos critérios de diagnóstico e à prevalência da doença no mundo.
\end{abstract}

DESCRITORES: Hipertensão. Pressão arterial.

\section{PRESSAO ARTERIAL. CONCEITO. VARIAÇŌES DA PRESSÃO ARTERIAL}

Os conhecimentos atuais sobre a pressão arterial (PA) baseiam-se na aplicação criteriosa dos princípios da hidrodinâmica ao sistema circulatório. As possíveis ressalvas a esta extensão da física para a compreensão da físiologia cardiovascular decorrem do fato de não ser o sistema circulatório composto por condutos rígidos, e nem o sangue pode ser considerado um fluido homogêneo com fluxo laminar. Ainda que com limitaçōes, esta aplicação da dinâmica dos fluídos contribuiu para o desenvolvimento da hemodinâmica e da físiopatologia cardiovascular 28,43,48.

A pressão arterial é aquela existente no interior das artérias e comunicada às suas paredes. Pela equação de Poiseuille-Hagen, a PA pode ser calculada pelo produto da resistência vascular periférica total pelo débito cardíaco 28,43 . Assim, devido a esta proporcionalidade, todos os fatores que alteram estas duas variáveis podem alterar a PA. Dentre aqueles que modificam o débito cardíaco, deve-se mencionar as alteraçóes da volemia, da contratilidade do miocárdio e da freqüência cardíaca. Já a regulação da resistência vascular periférica depende do complexo mecanismo de regulação da resistência das arteríolas, no qual atuam de modo interrelacionado: o balanço de eletrólitos, especialmente do sódio, do potássio e do cálcio; o sistema renina-angiotensina-aldosterona; os baroceptores do seio carotídeo, do arco abrtico e do átrio direito; neurotransmissores como a epinefrina e a norepinefrina; e hormônios de diversas glândulas (hormônio antidiurético, ACTH, cortisol, prostaglandinas, sistema calicreína-cinina, hormônio natriurético renal, dentre outros) ${ }^{28,43}$.

A PA varia entre um valor máximo durante a sístole (pressão arterial sistólica - PAS) e um mínimo na diástole (pressão arterial diastólica PAD).

\section{HIPERTENSÃO ARTERIAL. CONCEITO. IMPLI- CAÇOES DO CONCEITO}

A Organização Mundial da Saúde ${ }^{49}$ definiu, em 1978, a hipertensão arterial como sendo "uma doença caracterizada por uma elevação crônica da pressão arterial sistólica e/ou pressão arterial diatolica".

A conceituação utilizada implica a consideração dos seguintes aspectos:

a) o diagnóstico da doença depende de Medidas de pressão arterial, que apresentam uma Variabilidade;

b) estas medidas são consideradas Elevadas com relação a um determinado padrão de Normalidade;

c) esta elevação é dita Crônica, sem maiores especificações de tempo.

\section{MEDIÇÃO DA PRESSÃO ARTERIAL. VARIA- BILIDADE DA MEDIDA}

A primeira determinação da PA foi feita por via intra-arterial em animal no ano de 1730 por Hales na Inglaterra ${ }^{2}$. Hoje em dia, esta técnica invasiva se destina no ser humano unicamente à investigaçăo49. As medidas usuais de PA, quer para uso clínico, quer para uso epidemiológico, dependem de aparelhos portáteis que utilizam o método indireto de medida, inventado por Scipione Riva Rocci em 1898, baseado na oclusão do fluxo da artéria braquial por um manguito pneumático ligado a um manômetro de mercưrio ${ }^{2,49}$. Este manômetro pode ser do tipo aneróide ou de coluna de mercúrio ${ }^{49}$.

\footnotetext{
* Parte da Tese de Doutorado apresentada à Faculdade de Medicina da Universidade de São Paulo, em 1989, intitulada "Prevaléncia de hipertensão arterial no Município de Araraquara, SP, Brasil, 1987.

* Departamento de Epidemiologia da Faculdade de Saúde Pública da Universidade de São Paulo - Av. Dr. Amaldo, 715 — 01255 - São Paulo, SP - Brasil.
} 
Em 1905, Nicolai Korotkoff mostrou ser possível ouvir os rúdos produzidos quando o manômetro era esvaziado; a ausculta dos mesmos, com o auxílio de um estetoscópio aplicado sobre a artéria braquial durante a deflação do manguito pneumático, permitiu a caracterização de "fases" destes ruídos, cujo início e fim podem ser usados para avaliar a PAS e a PAD 2,40. Assim, considerase hoje que o primeiro aparecimento dos sons ao se fazer a deflação do manguito constitui uma boa aproximação da $\mathrm{PAS}^{49}$. O ponto em que os sons desaparecem (fase V) é usualmente tomado como a $P A D^{40,49}$, por ser este ponto de identificação mais confiável e mais reprodutível $2,46,49$. Discute-se se a fase IV (abafamento dos sons) deva ser adotada como uma melhor aproximação da PAD, mas, em geral esta fase so tem sido usada para tal fim na gravide $Z^{49}$, ou em crianças, quando os rúdos podem se prolongar até o zero da escala ${ }^{40,49}$.

Como toda medida, a PA pode variar em função de flutuaçర̋es verdadeiras ou aparentes ${ }^{10}$. As primeiras se devem à variaçăo intraindividual, quer durante as 24 horas, quer durante o decorrer de períodos mais longos de tempo, em função de estímulos fisiológicos intrínsecos (sono, febre, exercício, dor, por exemplo), ou ambientais (frio, calor, ruído, dentre outros) ${ }^{10,39,49}$. Devido a esta variabilidade, discute-se a necessidade da medição da PA em condiçōes de repouso ("medidas basais") ${ }^{26}$ ou mesmo a motorização da PA nas 24 horas $^{49}$ mas, para fins práticos, são as medidas "casuais" as utilizadas ${ }^{39,49}$.

As flutuaçôes aparentes são erros de medida que podem estar relacionados $10,20,32,39,40,49$ :

- com o aparelho: por má conservação, descalibração, vazamentos, entre outros;

- com o observador: hipoacusia, uso do manguito inapropriado ao tamanho do braço, ou mal colocado; compressão da artéria braquial pela campânula do estetoscópio; escolha de dígitos terminais preferenciais, por vícios adquiridos de longa data e insuficiência de treinamento; esvaziamento muito rápido ou muito lento do manguito, dentre outros erros de técnicas;

- com o paciente: muito embora nem todos sejam per se erros de medida, já que decorrem de alteraçōes verificáveis no paciente, o potencial para vício deriva de não se ter em conta a existência das seguintes condiçбes, dentre outras:

- obesidade (sem a devida correção do tamanho do manguito) ${ }^{39 / 40}$

- presença de arritmias cardíacas ${ }^{49}$

- existência de artérias muito rígidas em idosos, uma condição às vezes referida como "pseudohipertensão" 9, 46

- uso de drogas vasodilatadoras, betabloqueadores e inibidores adrenérgicos ${ }^{49}$

- uso de estimulantes, em particular fumo e café 49

- tensão relacionada à medida ("hipertensão do avental branco") ${ }^{34}$

- exercício físico ou alimentação recente ${ }^{39,40}$

Em função da variabilidade da medida, a maioria dos estudos epidemiológicos, 10,39,40,49 embora dependa de medidas "casuais", tenta reduzir a ocorrência de vicios de aferição mediante o uso de:

- técnicas padronizadas para o treinamento do observador, com a confecção de protocolos detalhados de aferição da PA;

- emprego de esfigmomanômetros modificados, automáticos ou não, que possibilitem reduzir o vício do observador (ex.: random-zero, ou o da London School of Hygiene, com ou sem modificaçoes);

- manutenção e calibração freqüentes dos esfigmomanômetros, qualquer que seja o tipo (aneróide ou de mercúrio).

Como toda medida, a PA pode apresentar o fenômeno da regressão à média, devido a erro de medida ou à variabilidade fisiológica ${ }^{10,41}$. Assim, ao reexaminar um determinado entrevistado, a PA pode baixar em função de flutuaçðes casuais e assumir uma posição mais próxima da média verdadeira. Isto tem colocado em debate o número de medidas que devam ser feitas numa mesma ocasião bem como ao longo de um determinado período de tempo antes de se considerar que haja uma elevaçăoo "crônica" da pressão. Assim, a Organização Mundial da Saúde preconiza que, exceto em emergências, se façam ao menos três medidas em duas ocasižes diferentes, sob condiçбes controladas, isto é, de acordo com um protocolo para minimizar vícios de aferição, antes de se considerar um paciente como hipertenso ${ }^{49}$.

\section{PRESSÃO ARTERIAL "NORMAL"}

A definição de hipertensão arterial comporta, por outro lado, uma comparação com um padrão de normalidade. A variável pressão arterial, usualmente medida em $\mathrm{cm}$ ou $\mathrm{mm} \mathrm{Hg}$ ou em $\mathrm{kPa}$, é, contudo, uma variável contínua ${ }^{33}$. Excetuando populaçס̃es primitivas para as quais a PA, quer a sistólica quanto a diastólica, não se eleva com a idade, nas demais populações nota-se uma elevação com a idade, desde o nascimento ${ }^{10,30,31}$. Assim, nos diversos estudos de prevalência a PAS eleva-se continuamente com a idade, mostrando tendência a se estabilizar nos grupos etários mais velhos, sendo maior para homens do que para mulheres nos grupos mais jovens e, inversamente, maior nas mulheres mais velhas do que para homens da mesma idade $49,30,38$. Por sua vez, a PAD também se eleva com a idade até a quinta década, quando tende a atingir um máximo, depois estabilizando-se ou vindo declinar ${ }^{30,38,49}$.

Assim sendo, não existe um critério de corte que possa ser decorrente da distribuição da variável pressão nas populaçত̃es ${ }^{34,49}$. Qualquer definição de "normalidade" ou de "anormalidade" é arbitrária 
e adota critérios operacionais que dependem do conhecimento do risco de morbimortalidade por complicações da doença por estudos longitudinais e por investigaçz̃es da eficácia da intervenção médica (farmacologica ou não) sobre o prognóstico ${ }^{10}$.

\section{COMPLICAÇŐES DA HIPERTENSÃO ARTERIAL. O IMPACTO DO TRATAMENTO}

As complicações da HA derivam das modificaçōes anatômicas e fisiológicas decorrentes do regime de pressão a que estão submetidas as câmaras cardíacas e, também, da aceleração do processo aterosclerótico ${ }^{13,16}$. A HA atua sinergicamente com outros fatores de risco aterogênicos como a hiperlipidemia, o diabetes mellitus, a obesidade, o sedentarismo e o hábito de fumar, dentre outros ${ }^{15}$.

As lesб̃es conseqüentes à $\mathrm{HA}$ relacionam-se com o nível da PA. Tanto a elevação da PAS isolada (HA sistólica isolada), quanto a elevação da PAS e/ou da PAD são importantes ${ }^{10}$. Estas lesర̃es consistem basicamente de espessamento arteriolar e, nas fases complicadas, da formação de ateromas, de necrose arteriolar, trombose intravascular, formação de aneurismas em grandes vasos e de microaneurismas das artérias intracerebrais (aneurismas de Charcot-Bouchard)2, 13.

As alterações morfológicas e fisiológicas são mais freqüentemente observadas nos seguintes orgãos: coração (hipertrofia ventricular esquerda, insuficiência cardíaca ${ }^{19}$, doença coronária ${ }^{17}$ ), encéfalo (doença cerebrovascular hemorrágica ou isquêmica, encefalopatia hipertensiva), rim (nefrosclerose, insuficiência renal crônica), retina (retinopatia hipertensiva), e vasos (aneurismas de aorta, dissecantes ou não; aneurismas de outros vasos; obstrução arterial crônica) ${ }^{2}, 13,49$.

Os estudos longitudinais norte-americanos têm mostrado um papel mais relevante da HA para a ocorrência de hemorragia intracerebral e de hemorragia subaracnóide ${ }^{418}$ do que para outras complicações neurológicas ou de outros órgãos. E, para estas complicaç̃es neurológicas hemorrágicas há maior incidência delas em hipertensos de raça ne$\operatorname{gra}^{2}, 10,42$.

Há hoje evidências inequívocas de que a morbimortalidade cardiovascular pode ser reduzida com a intervenção sobre o curso clínico da doença mediante o emprego de drogas anti-hipertensivas $^{10,14}$.

$\mathrm{Na}$ opinião de Institutos Nacionais de Saúde do governo norte-americano ${ }^{14}$, a redução da PA com drogas é seguramente eficaz para reduzir a morbimortalidade dos pacientes com PAD igual ou maior a $105 \mathrm{~mm} \mathrm{Hg}$. Segundo esses órgãos, nos hipertensos com PAD entre 90 e $104 \mathrm{~mm} \mathrm{Hg}$ ocorre também benefício relevante, representado por redução da mortalidade por todas as causas e proteção com relação à incidência de doenças cerebrovasculares, doença coronária e da progressão a formas mais graves de hipertenção arterial ${ }^{14}$. O impacto é mais notório para a doença cerebrovascular do que para outras complicações, já que para a doença coronária a redução da mortalidade tem sido não significante do ponto de vista estatístico ${ }^{42}$.

Estas evidências são de extrema importância para a definição de critério de corte e para a instituição de programas de detecção, tratamento e controle da $\mathrm{HA}^{10,11,49}$. Nos últimos anos, tem-se enfatizado a necessidade do controle da $\mathrm{HA}$ leve e da HA sistólica isolada em idosos ${ }^{20}$.

\section{CRITÉRIOS DE DEFINIÇÃO DA HIPERTENSÃO ARTERRIAL}

\section{Organização Mundial da Saúde (OMS) 19784}

No ano de 1978, um Comitê de Peritos da OMS definiu como necessárias, para se considerar um adulto como hipertenso, ao menos três medidas de PA feitas em ao menos duas ocasiōes diferentes, sob condições padronizadas, exceto em casos de emergência.

Os níveis de corte deste critério utilizam para a PAS a fase I de Korotkoff, e para a PAD, a fase V. São os seguintes os níveis de corte:

- normotensos: PAS $<140 \mathrm{~mm} \mathrm{Hg}$ e PAD $<90$ $\mathrm{mmHg}$

- hipertensos: PAS $\geq 160 \mathrm{~mm} \mathrm{Hg}$ e/ou PAD $\geq$ $95 \mathrm{~mm} \mathrm{Hg}$

- hipertensos "borderline" (ou "limítrofes"): PAD $>90$ e $<95 \mathrm{~mm} \mathrm{Hg} \mathrm{e/ou} \mathrm{PAS}>140$ e $<160 \mathrm{~mm}$ Hg.

Neste documento oficial não eram definidos os limites inferiores da idade adulta, nem os níveis normais de pressão arterial em crianças.

Comitê Norte-Americano de Deteç̧ão, Avaliação e Tratamento de Hipertensão Arterial - Joint National Committee for the Detection, Evaluation and Treatment of High Blood Pressure - JNC -IV - 1988'4

O Programa Nacional sobre a Hipertensão Arterial do Governo dos Estados Unidos divulgou em maio de 1988 o quarto relatório do Comitê de Avaliação e Tratamento da Hipertensão Arterial, que definiu a forma de se efetuar o diagnóstico e os critérios de corte.

Para que um adulto (de 18 anos ou mais) seja diagnosticado clinicamente como hipertenso, o Comitê preconiza duas ou mais leituras por visita, em ao menos duas visitas subseqüentes; tais leituras devem ser feitas com técnica padronizada; toma-se a média das leituras. O valor utilizado para a PAD é a fase V. Os critérios de corte são os seguintes:

- HA leve: PAD 90-104 mm Hg 
— HA moderada: PAD 105-114 mm Hg

- HA grave (severa): PAD $\geq 115 \mathrm{~mm} \mathrm{Hg}$

- HA sistólica isolada: PAS $\geq 160 \mathrm{~mm} \mathrm{Hg}$ e

$\mathrm{PAD}<90 \mathrm{~mm} \mathrm{Hg}$

São considerados normotensos apenas aqueles indivíduos com PAD inferior a $85 \mathrm{~mm} \mathrm{Hg}$ e com PAS inferior a $140 \mathrm{~mm} \mathrm{Hg}$. Os demais indivíduos, não abrangidos nos itens anteriores, são considerados de risco aumentado e devem ter a sua PA monitorada no decorrer do tempo ("high normal" ou hipertensão borderline sistólica isolada).

\section{CLASSIFICAÇÃO ETIOLÓGICA DA HIPERTENSÃO ARTERIAL}

A hipertensão arterial pode ser também classificada pela sua etiologia. Acredita-se que 95 e 99\% dos casos são de hipertensão primária ou essencial, para a qual nāo existe causa orgânica evidente ${ }^{49}$. Para os demais casos, a hipertensão é secundária à administração de drogas (contraceptivos orais, hormônios da suprarrenal, dentre outras); gravidez; doença cardiovascular como coarctação da aorta, doença renal; doença das glândulas suprarrenais (córtex ou medula) ${ }^{49}$.

\section{CAUSAS DA HIPERTENSÃO PRIMÁRIA}

E preciso distingüir, em primeiro lugar, a importância relativa da hereditariedade e dos fatores ambientais para a gênese da hipertensão primária.

Em relação à Herança da doença, as evidências apontam para a agregação familiar dos $\operatorname{casos}^{30}$, ao menos em parentes de primeiro grau, embora existam vícios de medida (por uma informação mais freqüente dos hipertensos de que seus ascendentes ou descendentes são hipertensos) e vícios de confusão (porque as pessoas podem ter não só em comum o mesmo patrimônio genético mas também a mesma dieta e o mesmo ambiente de vida $)^{30,31}$. Na literatura, a forma de herança foi motivo da controvérsia de Platt ${ }^{36}$ e Pickering ${ }^{34}$; o primeiro propugnava que a base da herança era um gene único, com dominância incompleta, que determinaria a "qualidade" de ser ou não hipertensos; o segundo afirmava que a herança seria poligênica, influindo sobre o nível ("quantitativo") da pressão arterial.

Estudos de diversos autores em gêmeos mostram maior semelhança da PA entre os monozigóticos que entre os dizigoticos. Entre pais que adotaram filhos e estes últimos, não existe correlação entre os níveis de PA, mostrando a importância do patrimônio genético ${ }^{49}$.

A inexistência de níveis de corte claros, e ainda, de duas subpopulaç̋es (uma, de normotensos, e outra, de hipertensos) na população geral, favorece a hipótese de Pickering $30,31,34$. Acredita-se hoje que a herança seja muito mais permissiva do que determinativa ${ }^{31}$.

Discute-se, além disto, como esta predisposição genética atuaria, se mediada ou não pela incapacidade de lidar com sobrecargas de sódio, a exemplo do modelo animal de $\mathrm{Dahl}^{31}$, ou ainda, por anormalidade, na função renal ou simpática ${ }^{50}$.

Um outro ponto a considerar em relação à etiologia da HA primária é o que se refere ao Grupo Étnico das populações estudadas. A prevalência de hipertensão arterial é mais alta em negros do que em brancos nos Estados Unidos da América, apresentando os primeiros complicaçōes com maior freqüência, sobretudo doenças cerebrovasculares e insuficiência cardíaca congestiva ${ }^{8,10,38}$. No Brasil, a prevalência de hipertensão arterial tem se mostrado maior em negros do que em mulatos, e nestes, maior do que em brancos 6,37 .

Em outros grupos étnicos, a prevalência é também bastante alta, como por exemplo entre os japoneses de Honshu e entre coreanos ${ }^{30,31}$. A análise desta característica envolve a consideração do patrimônio genético semelhante, e também de condições sociais que podem confundir a análise como o "stress" psicossocial, a ocorrência de pessoas de menor renda, mais baixa escolaridade formal, maior freqüência de obesidade e acessibilidade à assistência médica, dentre outras $8,30,38$.

Em ao menos vinte populaçōes primitivas, compostas de aborígenes dos diversos continentes, temse verificado que a PAS e a PAD năo se elevam com a idade, nem existe a doença "hipertensão arterial"30,31. Esta passa a ocorrer quando estes indígenas se aculturam, com a mudança de seus hábitos de vida (maior sedentarismo, excessiva ingestão de sodio, obesidade) ${ }^{10,30,31}$.

Quanto à Idade, a PA é, na maioria das populaçőes, mais baixa no nascimento, crescendo subseqüentemente durante toda a vida do indivíduo, para a PAS continuamente, e para a PAD subindo até a quinta década para homens, e até a sexta década para mulheres, declinando daí por diante ${ }^{49}$. Em homens a prevalência tende a ser discretamente maior para a população adulta, ao menos nas idades inferiores a 45 anos ${ }^{8,38}$.

Embora não existam estudos internacionais com metodologia padronizada para a avaliação da influência da Classe Social, tem-se notado uma relação inversa da prevalência de hipertensão arterial com a escolaridade formal, a posição social na ocupação e a renda familiar. Isto é, quanto mais privilegiado o indivíduo na sociedade, menor a prevalếncia de hipertensão arterial $1^{6,8,21,38}$.

Por outro lado, há outras evidências que estas mesmas variáveis (renda, ocupaçăo, escolaridade) estão associadas de perto com a situação urbana da moradia do entrevistado a ao estresse psicossocial, 
ainda que este termo não seja adequadamente definido $^{31}$. Outros estudos mostram também que a migração de indivíduos de áreas rurais para a zona urbana do mesmo País ou de outro é fator importante para a ocorrência de elevação da PA e aumento da prevalência de hipertensão arterial ${ }^{24,31}$. Isto foi verificado nos Tokelau na Oceania que migraram para a Nova Zelândia, nos japoneses que migraram para o Havaí e para o território continental dos Estados Unidos ${ }^{10}$, e para os migrantes internos procedentes da zona rural no Estado do Rio Grande do Sul e que passaram a residir na zona urbana do Grande Porto Alegre ${ }^{24}$.

E extremamente rica a literatura sobré a ingestão de Eletrólitos na dieta, sobretudo o Sódio no sal de cozinha ${ }^{45,49}$. Os dados existentes envolvem consideraçōes antropológicas que indicam que a adição de sal ao alimento é recente na história da humanidade, relacionada à necessidade de conservação do mesmo, e não relacionada às necessidades dietéticas do eletrólito ${ }^{31,45}$. Por outro lado, as populações primitivas que não apresentam hipertensão arterial ingerem também baixo conteúdo de sódio na dieta, mostrando-se sempre com PAs baixas para a idade e sem ascensão da PA com a idade ${ }^{31,45}$. Em diversas populaçð̄es tem-se mostrado a correlação da ingestão de sódio com a prevalência de hipertensão arterial ${ }^{31}$. Problema adicional para estas análises diz respeito à metodologia da avaliação da ingestão diária de sódio ${ }^{45}$. Por outro lado, a forma de atuação do sódio, e também de eletrólitos relacionados como potássio e cálcio, se daria ao nível intracelular, no mecanismo da bomba de sódio ${ }^{45}$. Tem-se descrito, também, que a ingestão grande de sódio está freqüentemente associada à baixa ingestão de potássio, sendo que este último poderia, eventualmente, ter um efeito protetor sobre a pressão arteriali ${ }^{31}$. Outras evidências a favor do papel fundamental desempenhado pela ingestão de sódio dizem respeito ao benefício das dietas com baixo conteúdo de sódio para o tratamento da hipertensão já instalada ${ }^{14}$.

Outro corpo de evidências favorece a Obesidade como importante fator de risco para o desenvolvimento da hipertensão primária no adulto 27,50 . Os estudos longitudinais e transversais norteamericanos mostram haver correlação entre a PA e a massa corpórea, avaliada por qualquer método (índice de massa como Quetelet ou peso acima do ideal) ${ }^{27,50}$, havendo indicações de que não se trata de um artefato de medida, decorrente do uso de manguitos inadequados para pessoas obesas $27,50.0$ risco aumenta quanto maior o ganho de peso ${ }^{27}$. Por outro lado, a redução de peso em pessoas que já eram hipertensas se associa com a queda da pressão arterial ${ }^{49}$. O Quetelet está associado com a PAS e a PAD 6 .

Quanto ao uso de Álcool tem-se mostrado que um consumo excessivo de álcool está associado com maior prevalência de hipertensão, tanto em estu- dos transversais, quanto em estudos de coorte 2,50 . Discute-se a via pela qual o álcool poderia aumentar a PA de um individuo, embora este tenha uma ação direta sobre o músculo cardíaco; há evidências também de uma ação indireta sobre o metabolismo de cálcio ${ }^{7}$. Em doses moderadas ou baixas, o consumo de álcool tem-se associado com prevalências mais baixas de hipertensão arterial e com mortalidade menor de doenças cardiovasculares, sendo que uma das hipoteses poderia ser a redução do estresse ou ainda alterações do metabolismo de cálcio e do colesterol (diminuição do LDL colesterol e elevação do HDL colesterol) ${ }^{7,10}$. E importante, entretanto, que se considere sempre a possibilidade de vícios de aferição da ingestão de álcool já que nestes estudos a informação é colhida do próprio entrevistado ${ }^{7}$

Em relação ao uso de Fumo, diversos estudos na literatura têm falhado em mostrar associaçōes consistentes entre o hábito de fumar e a prevalência de hipertensão arterial, quando se controlam variáveis de confusåo como a obesidade, a idade e o sexo 231,50 . Sabe-se, contudo, que os fumantes têm maior chance de evoluir para formas malignas da doença ${ }^{3} \mathrm{e}$, também, de morrerem mais freqüentemente de morte súbita cardíaca e por doença coronária ${ }^{3}$. Evidentemente, os riscos do uso de fumo e de álcool e aquele da obesidade são sinérgicos ${ }^{10}$.

O uso de Anticoncepcionais Orais está, por sua vez, associado ao desenvolvimento de hipertensão, ainda que transitória, que cessa 3 a 6 meses após a interrupção da droga ${ }^{31}$. Este fato ocorre mais freqüentemente em mulheres após os 35 anos. Embora se trate de uma hipertensão secundária à droga, pode-se discutir se a ingestão de contraceptivos não se constituiria em desencadeante de hipertensão em pessoas predispostas geneticamente.

\section{PREVALÊNCIA DE HIPERTENSÃO ARTERIAL}

A prevalência de HA varia bastante de país para país, não só em função de diferenças reais entre as populações mas também em função dos diferentes critérios de corte utilizados, assim como da inclusão, entre os hipertensos, de pessoas tratadas e controladas. Assim, nos Estados Unidos, para os anos de 1971-1975, em adultos de 25-74 anos, de ambos os sexos, com o critério da $\mathrm{OMS}^{49}$, a prevalência foi de $18,0 \%{ }^{38}$, e para os anos de 1976-1980, de $17,7 \%^{8}$. Nestes estudos incluíram-se os controlados. A prevalência de hipertensão "borderline" nos mesmos estudos foi, respectivamente, de $17,1 \%^{38}$ e $12,0 \%{ }^{8}$. No estudo de $1976-1980$, utilizando-se o critério do Joint National Committee IV,32 a prevalência de HA definida sobe para $29,7 \%{ }^{8}$.

Deve-se mencionar no Brasil estudos transversais que abrangeram um Estado (Rio Grande do Sul, $1978)^{6,21}$ e um município (Volta Redonda, 1979/ 1980) 22 . Para o Rio Grande do Sul, a prevalência da HA na população de 20-74 anos, de ambos os 
sexos, usando-se como critério o da OMS ${ }^{49}$, e ar incluindo os controlados, foi de $11,3 \%^{6,21}$. Já em Volta Redonda, RJ, a prevalência de $\mathrm{HA}$ para o mesmo grupo etário e com o mesmo critério, foi de $10,1 \% 22$.

Outros estudos no País buscaram avaliar a prevalência de $\mathrm{HA}$ em diversos grupos sociais ${ }^{5}$, em comunidades menores ${ }^{5,12}$ e na população jovem ${ }^{1}$. Contudo, revestem-se de especial importância os estudos referentes à população trabalhadora urbana.

Assim, na Grande São Paulo, em estudo de 5.500 trabalhadores de 15 a 65 anos, de ambos os sexos, de dez subsetores da economia, observou-se uma prevalência de $18,1 \%$ para homens e de $6,6 \%$ para mulheres (hipertensão: PAD > $90 \mathrm{~mm} \mathrm{Hg}$ ) ${ }^{87}$. Tinham prevalências mais altas os trabalhadores dos subsetores de metalurgia, jornalismo, transporte e finanças ${ }^{37}$. Trata-se, neste estudo específico, de uma população selecionada, já que são excluídos aqueles hipertensos incapazes para o trabalho.

\section{O EMPREGO DE DADOS SECUNDÁRIOS PARA A AVALIAÇÃO DA IMPORTÂNCIA DA HI- PERTENSĀO ARTERIAL NA SOCIEDADE}

Os estudos de prevalência da HA são em geral extremamente caros e de abrangência restrita. A maioria das informaçōes rotineiras sobre a doença depende de dados secundários, dentre os quais cabe destacar as estatísticas de mortalidade, as estatísticas de morbidade hospitalar, além de informações sobre incapacitações temporárias ou permanentes decorrentes da $\mathrm{HA}$. Estes dados permitem ter uma idéia de magnitude da HA na sociedade.

Com relação à Mortalidade, deve-se ter em conta que, devido às disposiçőes internacionais de codificação da causa básica das declarações de óbito ${ }^{23}$, a enumeração das mortes devidas à doença hipertensiva é subestimada, dado que quando esta é mencionada junto com complicaçóes como a doença coronária ou a doença cerebrovascular, estas últimas são "preferidas" à hipertensão ${ }^{23}$. 0 exato número de pessoas que morrem com menção de HA na declaração de óbito só pode ser obtido pela metodologia de causas múltiplas ${ }^{23}$.

Conquanto com limitações, os estudos de mor- talidade por doenças cardiovasculares, com relação à sua magnitude e tendências, têm sido importantes nas duas últimas décadas, quer nos países desenvolvidos, quer no Brasil. A razão disto é que estes estudos foram os primeiros a assinalar um declínio da mortalidade por doença coronária e daquela por doenças cerebrovasculares, tanto nos Estados Unidos quanto em outros países industrializados $^{47}$ e também no Município de São Paulo ${ }^{25}$. Este declínio da mortalidade não pôde ser atribuído a artefatos ${ }^{25,47}$. Na medida que se trata de um fato real, isto permite que se discuta o modo como se pode estar atuando eficazmente sobre as doenças citadas, para as quais a HA é importante fator de risco ${ }^{45}$.

Quando se dispōem de estatísticas de Morbidade e de Custos (de internações hospitalares e/ou de atendimentos ambulatoriais), estes dados podem oferecer informaçōes indiretas sobre a prevalência da doença além de outras informações necessárias para o planejamento da assistência aos doentes de HA. A importância da HA como causa de atendimento dos serviços contratados da Previdência Social no Brasil, em 1985, foi avaliado por um grupo de pesquisadores da Escola Nacional de Saúde Pública ${ }^{35}$. Das internaçóes hospitalares da rede contratada, $2,66 \%$ o foram por doença hipertensiva; a elas poder-se-ia agregar aquelas por doença coronária $(1,48 \%)$, doenças da circulação pulmonar e por outras doenças do coração $(4,47 \%)$ e por doenças cerebrovasculares $(2,26 \%)$, já que em sua grande maioria se associam à $\mathrm{HA}$. $O$ mesmo estudo mostrou também uma alta letalidade hospitalar e alta proporção de gastos para o atendimento a essas patologias.

O custo alto de atendimento aos pacientes hipertensos foi também mostrado em hospital universitário do Rio de Janeiro ${ }^{44}$. Cada paciente hipertenso acarretava um custo hospitalar direto anual de US\$102, a maior parte ligada a consultas e internaçð̋es por complicações ${ }^{44}$.

As Incapacitaçōes săo outra fonte de informação com relação à magnitude da $\mathrm{HA}$ na sociedade. Em 1978, para o Brasil como um todo, o tempo médio de afastamento por HA era duas vezes o tempo médio de afastamento por todas as outras causas, isto é, 370 dias contra 178 , respectivamente. No mesmo ano, a HA era a principal causa de incapacidade definitiva ${ }^{6}$. 
LOLIO, C. A. de [The epidemiology of arterial hypertension]. Rev. Saúde públ., S.Paulo, 24:425-32, 1990.

ABSTRACT: A review of the knowledge of arterial hypertension, emphasizing the diagnostic criteria in use and the prevalence of the disease throughout the world, is presented.

KEYWORDS: Hypertension. Blood pressure.

\section{REFERENCIAS BIBLIOGRÁFICAS}

1. ALMEIDA, D. B. et al. Estudo comparativo de pressão arterial e da prevalência de hipertensão arterial em duas coortes sucessivas (1975-1976) de estudantes de 16 a 25 anos, Botucatu, SP, Brasil. Rev. Saúde públ., S. Paulo, 12: 497-505, 1979.

2. BEEVERS, D. G. \& MACGREGOR, G. A. Hypertension in practice. London, Martin Dunitz, 1987.

3. BLOXHAM, C. A. et al. Malignant hypertension and cigarette smoking. Brit. med, J., 1: 381-3, 1979.

4. BROTT, T. et al. Hypertension as a risk factor for spontaneous intracerebral hemorrhage. Stroke, 17: 1078-83, 1986.

5. CARVALHO, J. J. M. et al. Pressão arterial e grupos sociais. Estudo epidemiológico. Arq. bras. Cardiol., 40: $115.20,1983$.

6. COSTA, E. A. Magnitude da hipertensão arterial no Brasil. Ciênc. e Cult., 35: 1636-7, 1983.

7. CRIQUI, M. H. Alcohol and cardiovascular mortality. In: Kaplan, R. M. \& Criqui, M. H., eds. Behavioral epidemiology and disease prevention. New York, Plenum Press, 1985. p. 67-89.

8. DRIZD, T. et al. Blood pressure levels in person 18-74 years of age in 1976.80, and trends in blood pressure from 1960 to 1980 in the United States, Vital Hlth Statist. Ser. 11, (234) 1986.

9. FRANÇA, H. H. Pseudo-hipertensão. Arq. bras. Cardiol., 49: $67-8,1987$.

10. FRASER, G. E. Preventive cardiology. New York, Oxford University Press, 1986.

11. GIUMETTI, D. et al. Need to prevent and control highnormal and high blood pressure, particularly socalled "mild" hypertension: epidemiological and clinical data. Prev. Med., 14: 396-412, 1985.

12. HADDAD, N. Inquérito epidemiológico sobre cardiopatias crônicas em um bairro de Ribeirão Preto, São Paulo, Brasil. Arq. Hig. Saúde públ., 32/33: 27-77, $1967 / 1968$.

13. IGUCHI, M. de L. \& BRITO, T. de Hipertensão arterial: aspectos morfológicos. In: Chiaverini, R., ed. Doença hipertensiva: diagnóstico, etiopalogêneses, tratamento. Rio de Janeiro, Atheneu, 1980. p. 89 116.

14. JOINT National Committee on Detection, Evaluation and Treatment of High Blood Pressure; the 1988 report-4th report. Arch. int. Med., 148: 1023-38, 1988.

15. KANNEL, W. B. An overview of the risk factors for cardiovascular disease. In: Kaplan, N. M. \& Stamler, J., eds. Prevention of coronary heart disease: practical management of the risk factors. Philadelphia, W. B. Saunders, 1983. p. 1-19.

16. KANNEL, W. B. Hypertension: relationship with other risk factors. Drugs, 31 (Suppl.4): $1-11,1986$.

17. KANNEL, W. B. et al. Blood pressure and risk of coronary heart disease: the Framingham study. $D$ is. Chest, 56: 43-52, 1969.

18. KANNEL, W. B. et al. Epidemiologic sssessment of the role of blood pressure in stroke. J. Amer. med. Ass., 214: 301-10, 1970.

19. KANNEL, W. B. et al. Role of blood pressure in the development of congestive heart failure: the Framingham study. New Engl. J. Med., 287: 781-7, 1972.

20. KANNEL, W. B. et al. Perspective on systolic hypertension: the Framingham study. Circulation, 61: $1179.82,1982$

21. KLEIN, C. H. Hipertensão arterial em estratos geoeconômicos do Rio Grande do Sul. Rio de Janeiro, 1981. [Dissertação de Mestrado - Escola Nacional de Saúde Pública/FIOCRUZ].

22. KLEIN, C. H. et al. Inquérito epidemiológico sobre hipertensāo arterial em Volta Redonda, RJ. Cad. Saúde públ., Rio de Janeiro, 1: 58-70, 1985.

23. LAURENTI, R. Mortalidade por hipertensão arterial como causa básica. Ciênc. e Cult., 35: 1637-42, 1983 .

24. LEAL, M. C. et al. Migração intema e pressão arterial no Rio Grande do Sul. Cad. Saúde públ., Rio de Janeiro, 1: 207-19, 1985.

25. LOLIO, C. A. de et al. Decline in cardiovascular disease mortality in the city of S. Paulo, Brazil, 1970 
to 1983. Rev. Saúde públ., S. Paulo, 20: '454-64, 1986.

26. MAGALDI, C. \& ALMEIDA, D. B. Aspectos epidemiológicos e preventivos da doença hipertensiva. Arq. bras. Cardiol., 31: 341-7, 1978.

27. NEGRI, E. et al. Body weight and the prevalence of chronic diseases. J. Epidem. Community Hlth, 42: 24-9, 1988.

28. OIGMAN, W. Bases hemodinâmicas da hipertensão arterial. Arq. bras. Cardiol., 49: 303-8, 1987.

29. ORGANIZAÇÃO PAN-AMERICANA DA SAÚDE. A hipertensão arterial como problema de saúde comunitária: manual de normas operacionais para um programa de controle nos diferentes níveis de atenção. Brasília, Ministério da Saúde. Divisão Nacional de Doenças Crônico-Degenerativas, 1986. (Série PALTEX n 3).

30. PAGE, L. B. Epidemiologic evidence on the etiology of human hypertension and its possible prevention. Amer. Heart J., 91: 527.34, 1976.

31. PAGE, L. B. Epidemiology of hypertension. In: Genest, J. ed. Hypertension. $2^{\text {nd }}$ ed. New York, McGraw-Hill, 1983. p. 683-99.

32. PATTERSON, H. R. Sources of error in recording the blood pressure of patients with hypertension in general practice. Brit. med. J., 289: 1661-4, 1984.

33. PICKERING, G. Hypertension: definitions, natural histories and consequences. Amer. J. Med., 52: 57083, 1972.

34. PICKERING, T. G. et al. How common is white coat hypertension. J. Amer. med. Ass., 259: 225-8, 1988.

35. PINTO, C. B. et al. Morbidade hospitalar na rede contratada do INAMPS. RADIS-Dados, Rio de Janeiro, (12): 1-24, 1988.

36. PLATT, R. Heredity in hypertension. Lancet, 1: 899 904, 1963.

37. RIBEIRO, M. B. D. et al. Prevalência de hipertensão arterial na força de trabalho da Grande São Paulo: influência da idade, sexo e grupo étnico. Rev. Ass. med. bras., 28: 209-15, 1982.

38. ROBERTS, J. Hypertension in adults $25-74$ years of age, United States, 1971-1975. Vital Hith Statist. Ser. 11 (221), 1981.
39. ROSE, G. A. et al. A sphygmomanometer for epidemiologists. Lancet, 1: 296-300, 1964.

40. ROSE, G. A. et al. Métodos de encuesta sabre enfermedades cardiovasculares. $2^{\natural}$ ed. Ginebra, Organización Mundial de la Salud, 1982. (OMS-Serie Monografias, 56).

41. ROSNER, B. Screening for hipertension: some statistical observations. J. chron. Dis., 30: 7-18, 1977.

42. SACCO, R. L., et al. Sub-arachnoid and intracerebral haemorrhage: natural history, prognosis and precursive factors in the Framingham survey. Neurology, 34: 847-54, 1984.

43. SILVA, H. B. Fisiopatogênese da hipertensão arterial. In: Chiaverini, R., ed. Doença hipertensiva: diagnóstico, etiopatogênese, tratamento. Rio de Janeiro, Atheneu, 1980. p. 9-63.

44. SOUZA E SILVA, N. et al. Importância clínica dos custos hospitalares diretos em pacientes com hipertensão arterial num hospital universitário, Rio de Janeiro. Rev. Saúde públ., S. Paulo, 20: 293-302, 1986.

45. STAMLER, J. et al. High blood pressure: role in coronary heart disease and implications for prevention and control. Geneva, 1983. [Mimeografado].

46. STRONG, C. G. et al. Clinical examination and investigation of the hypertensive patient. In: Genest, J., ed. Hypertension. $2^{\text {nd. }}$ ed. New York, McGraw-Hill, 1983. p. 700-22.

47. UEMURA, K. \& PISA, Z. Recent trends in cardiovascular disease mortality in 27 industrialized countries. Wld Hlth Statist. Quart., 38: 142-62, 1985.

48. WALLACE, A. G. Pathophysiology of cardiovascular diseases. In: Smith, L. H. \& Thier, S. O., eds. $\mathrm{Pa}$ thophysiology: the biological principles of disease. Philadelphia, W. B. Saunders, 1981. p. 1072-264.

49. WORLD HEALTH ORGANIZATION. Expert Committee on Arterial Hypertension, Geneva, 1978. Report. Geneva, 1978. (Technical Report Series, 628).

50. WORLD HEALTH ORGANIZATION. Scientific Group on Primary Prevention of Essential Hypertension, Geneva, 1982. Report. Geneva, 1983. (Technical Report Series, 686).

Recebido para publicação em 10/11/1989 Aprovado para publicaşão em 17/4/1990 